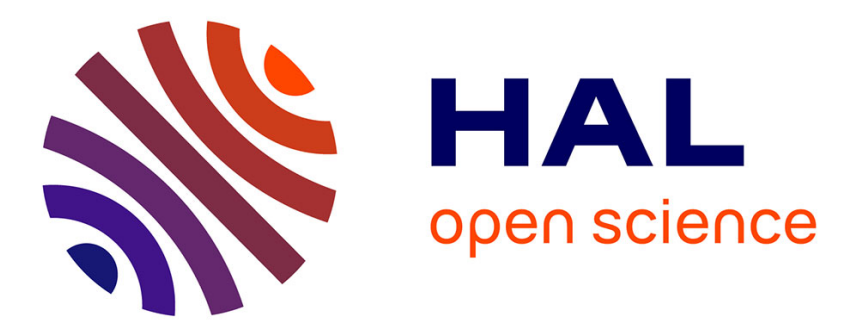

\title{
" Mattia Cavagna, La Vision de Tondale et ses versions françaises (XIIIe-XVe siècles). Contribution à l'étude de la littérature visionnaire latine et française
}

Estelle Doudet

\section{- To cite this version:}

Estelle Doudet. "Mattia Cavagna, La Vision de Tondale et ses versions françaises (XIIIe-XVe siècles). Contribution à l'étude de la littérature visionnaire latine et française. Cahiers de Recherches Médiévales et Humanistes = Journal of Medieval and Humanistic Studies, 2019. hal-02311497

\section{HAL Id: hal-02311497 \\ https://hal.science/hal-02311497}

Submitted on 11 Oct 2019

HAL is a multi-disciplinary open access archive for the deposit and dissemination of scientific research documents, whether they are published or not. The documents may come from teaching and research institutions in France or abroad, or from public or private research centers.
L'archive ouverte pluridisciplinaire HAL, est destinée au dépôt et à la diffusion de documents scientifiques de niveau recherche, publiés ou non, émanant des établissements d'enseignement et de recherche français ou étrangers, des laboratoires publics ou privés. 
Mattia Cavagna, La Vision de Tondale et ses versions françaises (XIII $-\mathrm{XV}^{e}$ siècles). Contribution à l'étude de la littérature visionnaire latine et française

\section{Estelle Doudet}

\section{(2) OpenEdition}

\section{Journals}

Electronic version

URL: http://journals.openedition.org/crm/15696

ISSN: 2273-0893

Publisher

Classiques Garnier

Electronic reference

Estelle Doudet, " Mattia Cavagna, La Vision de Tondale et ses versions françaises (XIIIe-XVe siècles).

Contribution à l'étude de la littérature visionnaire latine et française ", Cahiers de recherches médiévales et humanistes [Online], 2017, Online since 03 August 2019, connection on 04 August 2019. URL : http:// journals.openedition.org/crm/15696

This text was automatically generated on 4 August 2019

(c) Cahiers de recherches médiévales et humanistes 


\title{
Mattia Cavagna, La Vision de Tondale et ses versions françaises (XIII ${ }^{e}-X^{e}$ siècles). Contribution à l'étude de la littérature visionnaire latine et française
}

\author{
Estelle Doudet
}

\section{REFERENCES}

Mattia Cavagna, La Vision de Tondale et ses versions françaises (XIII ${ }^{e}-X V^{e}$ siècles). Contribution à l'étude de la littérature visionnaire latine et française, Paris, Champion (« Nouvelle Bibliothèque du Moyen Âge » 118), 2017, 674 p.

ISBN 978-2-7453-3152-6

1 L'ouvrage soumis à recension offre la première étude complète de la tradition française inspirée par La Vision de Tondale (Visio Tundali), l'un des textes majeurs de la littérature visionnaire occidentale. Le texte original et ses deux récritures latines ont en effet été traduits en seize langues et déclinés en de très nombreuses versions vernaculaires, du XIII e à la fin du $X^{e}$ siècle. Mattia Cavagna invite ici à découvrir un massif textuel quantitativement et qualitativement impressionnant : les onze adaptations en français de la Visio Tundali, conservées dans une trentaine de manuscrits. Seules six d'entre elles sont aujourd'hui accessibles par des éditions critiques, dont celles publiées par l'auteur en 2008 (La Vision de Tondale, les versions françaises de Jean de Vignay, David Aubert, Regnaud Le Queux, Paris, Champion, CFMA). Mais le lecteur francophone manquait jusqu'alors d'un guide sûr pour explorer ce corpus important, véritable point d'articulation entre la pensée eschatologique médiévale et les écritures du voyage vers l'au-delà illustrées par La Divine Comédie. L'ouvrage de synthèse de Mattia Cavagna comble donc un manque dans 
nos connaissances des imaginaires et des modes d'écriture visionnaires du Moyen Âge ; mais son apport va plus loin puisqu'il propose une relecture critique des hypothèses de Jacques Le Goff sur « l'invention » doctrinale du Purgatoire au XIII ${ }^{\mathrm{e}}$ siècle.

2 L'étude est fortement charpentée en deux parties. La première situe avec clarté les enjeux théologiques et littéraires de la Visio Tundali et de ses adaptations françaises. La seconde, plus brève, présente une description minutieuse des versions conservées et de leurs manuscrits, complétée par l'analyse des variations narratives apportées par chaque version. Deux annexes en fin d'ouvrage donnent à lire le prologue de la Visio originale latine et sa traduction en français moderne, ainsi que la liste des manuscrits latins qui ont soutenu la remarquable diffusion de ce texte dans la majorité des pays d'Europe. Les annexes sont suivies d'une bibliographie (p. 585-653) permettant de faire le tour de la question.

3 La présente recension s'attachera surtout à la première partie du livre, la plus longue et la plus riche d'informations. S'y déploie une analyse précise des principales caractéristiques du corpus, nourrie de réflexions sur l'état de la critique et de prises de position argumentées de l'auteur. La Visio Tundali est d'abord resituée dans son premier contexte de rédaction, en 1149, par le moine irlandais Frater Marcus, travaillant à Ratisbonne. Si elle s'est logiquement diffusée dans le sud de l'Allemagne dans un premier temps, la Visio s'inscrit dans la littérature irlandaise des echtrai et immrama, les navigations et autres voyages aventureux illustrés par la célèbre Navigation de saint Brendan. Elle est aussi modelée à partir de la nouvelle vision cistercienne du miles Christi et celle-ci permet de mieux saisir l'originalité du personnage de Tondale: différent des moines et clercs vertueux jusqu'alors protagonistes des visions eschatologiques, Tondale est un laïc, un chevalier qui a fait pendant sa vie fort mauvais usage des qualités qu'il a reçues de Dieu. La Visio fait en outre écho à des débats en cours sur la nature réelle ou symbolique des lieux de l'au-delà. Enfin, la confection rapide de deux récritures latines abrégées témoigne de l'immédiat impact du texte. Sa diffusion dans les espaces francophones a sans doute été facilitée par le fait que deux des remanieurs en latin, Hélinand de Froidmont puis Vincent de Beauvais, ont intégré leurs adaptations à des histoires universelles.

Pour expliquer le succès de la Visio pendant plus trois siècles, Mattia Cavagna s'attache ensuite à montrer l'originalité de ce texte. Issue de la puissante veine visionnaire qui se manifeste dans nombre d'apocryphes chrétiens, tels que l'Apocalypse de saint Paul ou le IV ${ }^{\mathrm{e}}$ livre d'Esdras, la Visio Tundali se singularise par plusieurs caractéristiques inédites. L'innovation qu'est le statut laïc donné à son narrateur a permis à ce personnage d'apparaître comme un exemple de l'humanité tout entière, mais aussi comme le miroir de tel ou tel prince destinataire; ce sera par exemple le cas de Charles le Téméraire, auquel est dédiée la version rédigée par le Bourguignon David Aubert au XV siècle. S'y ajoutent les circonstances particulières de la vision du chevalier : à la différence des stases mentales habituellement provoquées chez les hommes d'église par la maladie ou la pénitence, une mort temporaire frappe Tondale en plein repas pour le punir de ses fautes. Il est alors entraîné par un ange guide, marchant avec lui jusqu'au fond de l'Enfer puis montant jusqu'aux hauteurs inaccessibles du Paradis. Délaissant les extases immobiles de la littérature apocalyptique, la Visio Tundali et ses adaptations ont valorisé le mouvement dynamique, le dialogue vivant, la rencontre spectaculaire. Leur narrateur est l'un des premiers personnages de la littérature visionnaire à « plonger au fond du gouffre » pour observer la noirceur de Lucifer et la gloire de Dieu. 
5 Les onze variations rédigées $\mathrm{du}_{\mathrm{XIII}}{ }^{\mathrm{e}}$ au $\mathrm{XV}^{\mathrm{e}}$ siècle témoignent de la réception exceptionnelle de la Visio Tundali dans les régions d'expression française. Or ce succès n'a pas été sans ambiguïté. En effet, si l'intégration de l'histoire de Tondale dans le Miroir historial de Vincent de Beauvais a bien marqué un tournant dans la diffusion du texte, Mattia Cavagna démontre que cette valorisation s'est accompagnée de commentaires critiques mettant en jeu, voire en question l'authenticité du témoignage. Les questions de l'historicité et/ou de la fictionnalité de La Vision de Tondale ont donc été posées de conserve dès les premières adaptations françaises. En a découlé un possible « héritage littéraire» (p. 347) de la Vision, placé sous le signe de l'ambivalence. L'auteur propose de le détecter dans la floraison des Voies d'Enfer et de Paradis au tournant des XIII ${ }^{\mathrm{e}}$ et XIV ${ }^{\mathrm{e}}$ siècles, mais aussi dans la multiplication des voyages burlesques vers le ciel ou vers l'enfer jusqu'à la fin du XV $\mathrm{XV}^{\mathrm{e}}$ siècle. Du Songe d'Enfer de Raoul de Houdenc vers 1220 aux Purgatoire des mauvais maris et Enfer des mauvaises femmes remaniés sous le règne de Charles VIII, les enjeux moraux et pénitentiels de l'aventure visionnaire ont de fait souvent été infléchis en français par le rire parodique et par la récriture joyeuse de motifs devenus lieux communs. Il semble en fait assez délicat de découvrir l'influence de la seule Vision de Tondale sur de tels textes, aux sources d'évidence multiples. Mais si cette dernière argumentation ne convainc pas sur tous les points, elle a le grand mérite de souligner combien l'imaginaire visionnaire illustré par Tondale a travaillé en profondeur la culture littéraire de la fin du Moyen Âge.

6 Comme il a été indiqué en préambule, l'un des apports les plus décisifs du livre, qui intéressera les historiens comme les spécialistes de littérature, est certainement la relecture critique qu'il propose de l'invention doctrinale du Purgatoire au XIII siècle. Jacques Le Goff a insisté sur la reconnaissance officielle de ce lieu intermédiaire par le concile de Lyon en 1274 ; cependant, Mattia Cavagna précise à juste titre qu'à défaut du nom, la notion de purgatoire est déjà centrale dans la Vision de Tondale. Un tel lieu apparaît clairement sous la forme de l'enfer supérieur où se côtoient les « pas tout à fait mauvais » et les « pas tout à fait bons », réunis dans la même attente du salut (p. 275-306). Il sera donc judicieux à l'avenir de réintégrer à l'histoire des représentations de l'au-delà la source majeure qu'est la Vision de Tondale et ses versions en langue moderne, désormais accessibles dans des éditions récentes et éclairées par cet excellent ouvrage. 